Rapid Reviews COVID-19

\title{
Review 1: "Natural \\ deletions in the SARS-CoV- \\ 2 spike glycoprotein drive \\ antibody escape"
}

Mitnala Sasikala ${ }^{1}$, Ravikanth Vishnubotla ${ }^{2}$

${ }^{1}$ Director, Asian Institute of Gastroenterology, Molecular Biology and Biochemistry, India,

${ }^{2}$ Asian Institute of Gastroenterology

Published on: Apr 09, 2021

License: Creative Commons Attribution 4.0 International License (CC-BY 4.0). 


\section{$\underline{\text { RR:C19 Evidence Scale rating by reviewer: }}$}

- Reliable. The main study claims are generally justified by its methods and data. The results and conclusions are likely to be similar to the hypothetical ideal study. There are some minor caveats or limitations, but they would/do not change the major claims of the study. The study provides sufficient strength of evidence on its own that its main claims should be considered actionable, with some room for future revision.

$* * * * * * * * * * * * * * * * * * * * * * * * * * * * * * * * * * * * * * *$

\section{Review:}

Kevin R McCarthy et al have identified the mechanism by which SARS-CoV-2 evolves to become an endemic from a pandemic situation and persist as a respiratory virus in humans.

The authors first identified an immunocompromised patient who succumbed to infection after 74 days of Covid-19 diagnosis, despite treatment with Remdesivir and 2 infusions of convalescent serum. They identified 2 deletions in the $\mathrm{N}$ terminal domain (NTD) of spike protein upon sequencing of late time point clinical material. This finding encouraged the authors to probe patient metadata sequences retrieved from GISAID. Surprisingly, deletions akin to those seen in the immunocompromised patient from Pittsburg were seen in six of the eight patients. Additional searches involving 146,795 sequences in GISAID identified 1,108 sequences with deletions in the S gene, $90 \%$ of them located in four discrete sites called the Recurrent deletion regions 1-4 (RDRs) in NTD. Of these, RDRs 1and 3 were overwhelmingly from Europe indicating that selection favors specific deletions that circulate in certain geographies. The geographic distribution suggests that RDR variants with deletions transmit naturally in humans as seen in 2 patients from France, 4 patients from Ireland, 2 patients from Switzerland, and a few from the UK. While the RDR deletions were most frequent, they had no impact on the binding of antibodies. However, the authors demonstrate that deletions in RDRs 2 and 4 completely abolish the binding of antibodies to spike epitope by transfecting the plasmids with deletions into cell lines suggesting that these variants have altered antigenicity. The authors conclude that the deletions may have a confounding effect on vaccine candidates.

The study seems to be reliable as the authors sequenced the S gene from an immunocompromised patient and analyzed sequences data from already available data 
sets (though limited). However, the sequence data are not generated by the authors in a particular geographic area, as mentioned by themselves that the data is biased to the UK. The methods used are sufficient to suggest that deletions particularly in RDRs 2 and 4 alter the antigenicity, but are not robust. The functional efficacy of the antibody binding to the epitope needs to be demonstrated in various ways.

The manuscript confirms the previous work as the authors have analyzed already available sequence data from GISAID. It definitely contributes to the advancement of COVID-19 understanding as it has implications for the success or failure of the vaccine. The manuscript is worth publishing though limitations are not elaborated and ethics are not mentioned. The study is interesting and well-presented.

The authors have submitted the manuscript to a Preprint server. Hopefully, they have generated additional data in support of their claims and hence can be given an opportunity to publish the manuscript. 